\title{
Non-invasive prenatal testing for aneuploidy and beyond: challenges of responsible innovation in prenatal screening. Summary and recommendations
}

\author{
Wybo Dondorp ${ }^{\star, 1}$, Guido de Wert ${ }^{1}$, Yvonne Bombard ${ }^{2}$, Diana W Bianchi ${ }^{3}$, Carsten Bergmann ${ }^{4,5}$, Pascal Borry ${ }^{6}$, \\ Lyn S Chitty ${ }^{7}$, Florence Fellmann ${ }^{8}$, Francesca Forzano ${ }^{9}$, Alison Hall ${ }^{10}$, Lidewij Henneman ${ }^{11}$, Heidi C Howard ${ }^{12}$, \\ Anneke Lucassen ${ }^{13}$, Kelly Ormond ${ }^{14}$, Borut Peterlin ${ }^{15}$, Dragica Radojkovic ${ }^{16}$, Wolf Rogowski ${ }^{17}$, Maria Soller ${ }^{18}$, \\ Aad Tibben ${ }^{19}$, Lisbeth Tranebjærg ${ }^{20,21,22}$, Carla $\mathrm{G}$ van $\mathrm{El}^{11}$ and Martina C Cornel ${ }^{11}$ on behalf of the European \\ Society of Human Genetics (ESHG) and the American Society of Human Genetics (ASHG)
}

European Journal of Human Genetics advanced online publication, 1 April 2015; doi:10.1038/ejhg.2015.56

\begin{abstract}
INTRODUCTION
This paper contains a summary and the recommendations of a joint European Society of Human Genetics (ESHG)/American Society of Human Genetics (ASHG) position statement on responsible innovation in prenatal screening with non-invasive prenatal testing (NIPT). This statement was drafted by the Public and Professional Policy Committee of the ESHG and the Social Issues Committee of the ASHG, and endorsed by the boards of both societies in December 2014. The statement is also endorsed by the Human Genetics Society of Australasia, the Australasian Association of Clinical Geneticists, the British Society for Genetic Medicine, the Czech Medical Genetics Society, and the PHG Foundation (Cambridge, UK). The full document with extensive references is published separately. ${ }^{1}$
\end{abstract}

\section{SUMMARY OF THE ESHG/ASHG POSITION STATEMENT}

This ESHG/ASHG position statement takes as its starting point the internationally endorsed normative framework for prenatal screening, stressing that the aim of the practice should be to facilitate informed reproductive choices rather than preventing the birth of children with specific abnormalities, and that the benefits for those tested should clearly outweigh any harms. Moreover, when screening is offered as a public health programme, societal and justice aspects need to be taken into account. This includes possible consequences for other individuals and groups (including those living with the relevant conditions), as well as cost-effectiveness of publicly funded services.

In the past few years, professional bodies and policy authorities have recommended offering NIPT for common aneuploidies to women who belong to a higher risk group, either based on maternal age or a positive combined first trimester screening test (cFTS). With recent publications suggesting equally good test performance in lower-risk populations, and depending on the health care setting, different scenarios for NIPT-based screening for common autosomal aneuploidies are possible, including NIPT as an alternative first-tier test. The greater accuracy and lower invasive follow-up testing rate that can thus be achieved, has the potential of helping prenatal screening better achieve its aim, provided that balanced pre-test information and non-directive counseling are available as part of the screening offer. Concerns have been raised that as a result of these same features (greater accuracy and lower invasive follow-up testing rate), prenatal screening may increasingly be regarded both by professionals and pregnant women as a routine procedure that as such would not require much reflection. This may have the consequence that women or couples are insufficiently prepared for the possible eventual diagnosis of a fetus with a serious disorder. Avoiding such 'routinisation' effects may well be the greatest ethical challenge of NIPT-based prenatal screening.

Depending on the use of targeted or non-targeted analysis and on the level of resolution, NIPT for common autosomal aneuploidies may

${ }^{1}$ Department of Health, Ethics \& Society, Research Schools CAPHRI and GROW, Maastricht University, Maastricht, The Netherlands; ${ }^{2}$ Li Ka Shing Knowledge Institute of St. Michael's Hospital \& Institute of Health Policy, Management and Evaluation, Faculty of Medicine, University of Toronto,Toronto, ON, Canada; ${ }^{3}$ Department of Pediatrics, Obstetrics and Gynecology, Tufts University School of Medicine, Boston, MA, USA; ${ }^{4}$ Center for Human Genetics Bioscientia, Ingelheim, Germany; ${ }^{5}$ Department of Medicine, University Freiburg Medical Center, Freiburg, Germany; ${ }^{6}$ Department of Public Health and Primary Care, Centre for Biomedical Ethics and Law, Leuven University, Leuven, Belgium; ${ }^{7}$ Clinical and Molecular Genetics Unit, UCL Institute of Child Health, Great Ormond Street Hospital and UCLH NHS Foundations Trusts, London, UK; ${ }^{8}$ Service of Medical Genetics, University Hospital of Lausanne, Lausanne, Switzerland; ${ }^{9}$ Medical Genetics Unit, Ospedali Galliera, Genova, Italy; ${ }^{10} \mathrm{PHG}$ Foundation, Cambridge, UK; ${ }^{11}$ Section Community Genetics, Department of Clinical Genetics and EMGO Institute for Health and Care Research, VU University Medical Center, Amsterdam, The Netherlands; ${ }^{12}$ Centre for Research Ethics and Bioethics, Uppsala University, Uppsala, Sweden; ${ }^{13}$ Department of clinical ethics and law (CELS), University of Southampton and Wessex Clinical Genetic Service, Southampton, UK; ${ }^{14}$ Department of Genetics and Stanford Center for Biomedical Ethics, Stanford University School of Medicine, Stanford, CA, USA; ${ }^{15}$ Clinical Institute of Medical Genetics, Ljubljana University Medical Centre, Ljubljana, Slovenia; ${ }^{16}$ Laboratory for Molecular Biology, Institute of Molecular Genetics and Genetic Engineering (IMGGE), University of Belgrade, Belgrade, Serbia; ${ }^{17}$ Deutsches Forschungszentrum für Gesundheit und Umwelt, Helmholtz Zentrum, München, Germany; ${ }^{18}$ Division Clinical Genetics, University and Regional Laboratories Region Skåne, Lund University Hospital, Lund, Sweden; ${ }^{19}$ Department of Clinical Genetics, Leiden University Medical Center, Leiden, The Netherlands; ${ }^{20}$ Department of Audiology, Bispebjerg Hospital/Rigshospitalet, Copenhagen, Denmark; ${ }^{21}$ Department of Clinical Genetics/The Kennedy Center, University of Copenhagen, Copenhagen, Denmark; ${ }^{22}$ Institute of Cellular and Molecular Medicine, ICMM, University of Copenhagen, Copenhagen, Denmark

*Correspondence: Dr W Dondorp, Department of Health, Ethics \& Society, Research Schools CAPHRI and GROW, Maastricht University, PO Box 616, 6200 MD, Maastricht, The Netherlands. Tel: +31 43 3881712; Fax: +31 43 3670932; E-mail: w.dondorp@maastrichtuniversity.nl

Received 23 December 2014; revised 15 February 2015; accepted 19 February 2015 
lead to findings of abnormalities in other chromosomes, including submicroscopic abnormalities. This is not a new problem: such findings also emerge at follow-up testing after positive cFTS. However, at the NIPT stage, they precede decision making about invasive testing. This may entail putting the pregnancy at risk for confirming findings that not only have a low positive predictive value (because of their low frequency), but that, if confirmed, may still have highly uncertain implications for the expected phenotype. If enabling autonomous decision making is the aim of prenatal screening, then as much as reasonably possible, women or couples should be informed about the possibility of additional findings and their possible implications. They should also be given the opportunity to indicate whether or not they would want to receive information about clinically relevant findings beyond the scope of the screening offer.

A specific ethical issue related with NIPT is sex selection for nonmedical reasons. Depending on how the test is carried out, the scenario with NIPT as a first screening test might lead to information about fetal sex being available at an early stage in all screened pregnancies. Commercial companies offering NIPT currently also provide this information on an optional basis. There is a concern that some pregnant women and their partners may use this to have an abortion if the sex of the fetus does not match their preference. The best way to counteract improper use of information about fetal sex is to avoid it becoming available as part of the test results.

It is expected that in the coming years, it will become possible to use NIPT to screen for the same range of conditions that are currently tested for using karyotyping or microarray technologies at the follow-up stage, including sex-chromosomal and submicroscopic abnormalities. Commercial providers have already begun expanding their tests to include these conditions. However, this should not precede a careful assessment of the benefits and harms of doing so. Relevant aspects include: test accuracy, counseling challenges, women's preferences, the interests of the future child (see below) and misuse of information about fetal sex.

Given the publication of proof of principle analysis of the entire fetal genome from maternal plasma, it is expected to eventually become technically possible to turn NIPT-based screening into a comprehensive fetal genome scan, looking beyond chromosomal abnormalities to Mendelian disorders and genetic risk profiles for multifactorial diseases. Inevitably, this raises the question of what the scope of prenatal screening for fetal abnormalities should be. Reasons for not expanding prenatal screening beyond serious childhood disorders are the following. Firstly, unlimited choice may paradoxically undermine rather than serve or enhance reproductive autonomy. Secondly, given that expanded prenatal screening will reveal later onset disorders as well as abnormalities that might otherwise have remained undiagnosed, and that these findings will not always lead to the woman asking for an abortion, the interests and autonomy rights of the future child need to be taken into account. Bringing a child into the world with all the information from a full genome scan may lead to psychosocial harm and amount to a violation of his or her 'autonomy rights'.

Historically and ethically, prenatal screening for fetal abnormalities such as Down syndrome should be distinguished from prenatal screening for conditions relevant to a healthy outcome of the pregnancy for the mother and child. New developments including NIPT may increasingly lead to these types of prenatal screening running together. What should be avoided here, is to confuse women and couples with regard to what they are offered testing for, and what they can accept or decline, and on what basis. A further expected development is that more disorders will become treatable in utero. As a consequence, prenatal screening for those disorders will open up more options than only the choice between completion and termination of a pregnancy, including treatments that may benefit the health prospects of the future child. As a result, new ethical questions may arise for which the current ethical framework is inadequate. For instance: to what extent do these new preventative options create obligations for prospective parents?

In many countries, prenatal screening has in the past decades been offered to pregnant women in the form of national or regional population screening programmes. Whereas in the past, new screening technologies have been readily introduced in these programmes, the introduction of NIPT into clinical practice has until now been largely left to commercial laboratories offering their version of the test through individual practitioners and practices. Governments and public health authorities seem hesitant to be involved in this process. Given the importance of offering NIPT in a setting in which all relevant aspects (including information and counseling) are quality controlled, it is time that these actors take a more active role.

The notion that prenatal screening should be regarded a public health responsibility does not necessarily entail that all costs are paid from public money without any (co-)payment from those being tested. It is important to note that these are separate (though connected) issues. In light of the aim of providing options for reproductive choice, it might be argued that asking women to pay for prenatal screening increases the awareness that there is truly a choice to be made. On the other hand, depending on the height of the financial barrier, this may limit access to prenatal screening to those who are better off, which raises an issue of justice.

Finally, NIPT makes it possible in theory to offer prenatal testing for fetal abnormalities as a direct-to-consumer test. However, in order to maintain minimum quality standards, prenatal testing for fetal abnormalities should always be offered through health professionals with the expertise and training to provide the necessary pre-and posttest information and counseling.

\section{RECOMMENDATIONS}

1. NIPT offers improved accuracy when testing for common autosomal aneuploidies compared with existing tests such as cFTS. However, a positive NIPT result should not be regarded as a final diagnosis: false positives occur for a variety of reasons (including that the DNA sequenced is maternal and fetal in origin and that the fetal fraction derives from the placenta as well as the developing fetus). Thus women should be advised to have a positive result confirmed through diagnostic testing, preferably by amniocentesis, if they are considering a possible termination of pregnancy.

2. The better performance including lower invasive testing rate of NIPT-based screening should not lead to lowering standards for pre-test information and counseling. This is especially important in the light of the aim of providing pregnant women with meaningful options for reproductive choice. There should be specific attention paid to the information needs of women from other linguistic and cultural backgrounds or who are less health literate.

3. If NIPT is offered for a specific set of conditions (eg trisomies 21, 18 and 13), it may not be reasonably possible to avoid additional findings, such as other chromosomal anomalies or large scale insertions or deletions. As part of pre-test information, women and couples should be made aware of the possibility of such additional findings and the range of their implications. There should be a clear policy for dealing with such findings, as much as possible also taking account of pregnant women's wishes with regard to receiving or not receiving specific information. 
4. Expanding NIPT-based prenatal screening to also report on sexchromosomal abnormalities and microdeletions not only raises ethical concerns related to information and counseling challenges but also risks reversing the important reduction in invasive testing achieved with implementation of NIPT for aneuploidy, and is therefore currently not recommended.

5. Emerging opportunities for combining prenatal screening for fetal abnormalities with screening aimed at prevention may undermine adequate counseling by sending mixed messages. The objective of any prenatal screening activity should be made explicit and, as far as possible, forms of prenatal screening with different aims should be presented separately. If not physically possible, this separation should at least be made conceptually when providing the relevant information.

6. In countries where prenatal screening for fetal abnormalities is offered as a public health programme, governments and public health authorities should adopt an active role to ensure the responsible introduction of NIPT as a second or first-tier screening test for Down syndrome and other common autosomal aneuploidies. This entails ensuring quality control also extending to the non-laboratory aspects of NIPT-based prenatal screening (information and counseling), education of professionals, systematic evaluation of all aspects of the screening programme as well as promoting equity of access for all pregnant women within the confines of the available budget, and setting up a governance structure for responsible further innovation in prenatal screening.

7. Different scenarios for NIPT-based screening for common autosomal aneuploidies are possible, including NIPT as an alternative first-tier option. The inevitable trade-offs underlying those scenarios should not just be regarded as a matter of screening technology and health economics; the question is also how these trade-offs enable or impede meaningful reproductive choices and how they affect both the balance of benefits and burdens for pregnant women and their partners, and the screening goals and values acceptable to society.

8. In order to adequately evaluate prenatal screening practices, there is a need to further develop and validate measures of informed choice as well as interventions aimed at enabling informed choices. The transition to NIPT-based prenatal screening presents an opportunity to fill this gap in knowledge.

9. In the light of sequencing technologies becoming better and cheaper, there is an acute need for a proactive professional and societal debate about what the future scope of prenatal screening for fetal abnormalities should be. As argued in this document, there are strong ethical reasons for not expanding the scope of prenatal screening beyond serious congenital and childhood disorders.

10. The scenario in which prenatal screening would open up possibilities for fetal therapy in addition to autonomous reproductive choice raises fundamental questions about the relation between reproductive autonomy and parental responsibility that require an in depth proactive ethical analysis.

\section{CONFLICT OF INTEREST}

Employers of WD, GdW, $\mathrm{LH}, \mathrm{CvE}$ and $\mathrm{MC}$ received research grants for implementation and evaluation research on NIPT from The Netherlands Organisation for Health Research and Development (ZonMw) and The Netherlands Genomics Initiative. DB is a member of the Reproductive and Genetic Health Advisory Board for Illumina and receives an honorarium for this position. She also has a sponsored research grant from Illumina that is administered through Tufts Medical Center, Boston, MA, USA. CB is an employee of Bioscientia/ Sonic Healthcare, Ingelheim, while holding a part-time faculty appointment at the University of Freiburg, Germany. In addition, several of the authors work in health care settings in which NIPT is being offered.

\footnotetext{
1 Dondorp W, De Wert G, Bombard Y et al: on behalf of the European Society of Human Genetics (ESHG) and the American Society of Human Genetics (ASHG): non-invasive prenatal testing for aneuploidy and beyond: challenges of responsible innovation in prenatal screening. Eur J Hum Genet; e-pub ahead of print 18 March 2015; doi:10.1038/ejhg.2015.57
}

cc)(1) () $\odot$ This work is licensed under a Creative Commons Attribution-NonCommercial-NoDerivs 4.0 International License. The images or other third party material in this article are included in the article's Creative Commons license, unless indicated otherwise in the credit line; if the material is not included under the Creative Commons license, users will need to obtain permission from the license holder to reproduce the material. To view a copy of this license, visit http://creativecommons.org/licenses/by-nc-nd/4.0/ 